\title{
Front Matter: Volume 8148
}

, "Front Matter: Volume 8148," Proc. SPIE 8148, Solar Physics and Space Weather Instrumentation IV, 814801 (17 October 2011); doi:

10.1117/12.914131

SPIE Event: SPIE Optical Engineering + Applications, 2011, San Diego, California, United States 


\title{
PROCEEDINGS OF SPIE
}

\section{Solar Physics and Space Weather Instrumentation IV}

\author{
Silvano Fineschi \\ Judy Fennelly \\ Editors
}

21-24 August 2011

San Diego, California, United States

Sponsored and Published by

SPIE 
The papers included in this volume were part of the technical conference cited on the cover and title page. Papers were selected and subject to review by the editors and conference program committee. Some conference presentations may not be available for publication. The papers published in these proceedings reflect the work and thoughts of the authors and are published herein as submitted. The publisher is not responsible for the validity of the information or for any outcomes resulting from reliance thereon.

Please use the following format to cite material from this book:

Author(s), "Title of Paper," in Solar Physics and Space Weather Instrumentation IV, edited by Silvano Fineschi, Judy Fennelly, Proceedings of SPIE Vol. 8148 (SPIE, Bellingham, WA, 2011) Article CID Number.

ISSN 0277-786X

ISBN 9780819487582

Published by

SPIE

P.O. Box 10, Bellingham, Washington 98227-0010 USA

Telephone +1 3606763290 (Pacific Time) · Fax +1 3606471445

SPIE.org

Copyright (C) 2011, Society of Photo-Optical Instrumentation Engineers

Copying of material in this book for internal or personal use, or for the internal or personal use of specific clients, beyond the fair use provisions granted by the U.S. Copyright Law is authorized by SPIE subject to payment of copying fees. The Transactional Reporting Service base fee for this volume is $\$ 18.00$ per article (or portion thereof), which should be paid directly to the Copyright Clearance Center (CCC), 222 Rosewood Drive, Danvers, MA 01923. Payment may also be made electronically through CCC Online at copyright.com. Other copying for republication, resale, advertising or promotion, or any form of systematic or multiple reproduction of any material in this book is prohibited except with permission in writing from the publisher. The CCC fee code is 0277-786X/11/ \$18.00.

Printed in the United States of America.

Publication of record for individual papers is online in the SPIE Digital Library.

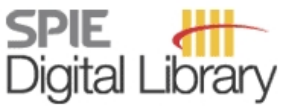

SPIEDigitalLibrary.org

Paper Numbering: Proceedings of SPIE follow an e-First publication model, with papers published first online and then in print and on CD-ROM. Papers are published as they are submitted and meet publication criteria. A unique, consistent, permanent citation identifier (CID) number is assigned to each article at the time of the first publication. Utilization of CIDs allows articles to be fully citable as soon as they are published online, and connects the same identifier to all online, print, and electronic versions of the publication. SPIE uses a six-digit CID article numbering system in which:

- The first four digits correspond to the SPIE volume number.

- The last two digits indicate publication order within the volume using a Base 36 numbering system employing both numerals and letters. These two-number sets start with 00, 01, 02, 03, 04, $05,06,07,08,09,0 A, 0 B \ldots 0 Z$, followed by 10-1Z, 20-2Z, etc.

The CID number appears on each page of the manuscript. The complete citation is used on the first page, and an abbreviated version on subsequent pages. Numbers in the index correspond to the last two digits of the six-digit CID number. 


\section{Contents}

ix Conference Committee

xi The little photometer that could: technical challenges and science results from the Kepler Mission (Plenary Paper) [8146-100]

J. M. Jenkins, NASA Ames Research Ctr. (United States); J. Dunnuck, Ball Aerospace \&

Technologies Corp. (United States)

xxiii Large Binocular Telescope Adaptive Optics System: new achievements and perspectives in adaptive optics (Plenary Paper) [8149-100]

S. Esposito, A. Riccardi, E. Pinna, A. Puglisi, F. Quirós-Pacheco, C. Arcidiacono, M. Xompero, R. Briguglio, G. Agapito, L. Busoni, L. Fini, J. Argomedo, A. Gherardi, INAF - Osservatorio Astrofisico di Arcetri (Italy); G. Brusa, D. Miller, J. C. Guerra, Large Binocular Telescope Observatory, The Univ. of Arizona (United States); P. Stefanini, P. Salinari, INAF - Osservatorio Astrofisico di Arcetri (Italy)

\section{SESSION 1 SPACE WEATHER INSTRUMENTATION}

814802 A collimator for measurements of the loss cone flux of energetic electrons [8148-01] J. D. Sullivan, Boston College (United States); C. W. Parker, NCR Research Asssociate (United States)

814803 The Energetic Particle Telescope (EPT) concept and performances [8148-02] M. Cyamukungu, G. Grégoire, Ctr. for Space Radiations (Belgium)

814804 Characterization of sensitivity degradation seen from the UV to NIR by RAIDS on the International Space Station [8148-03]

A. W. Stephan, U.S. Naval Research Lab. (United States); A. B. Christensen, The Aerospace Corp. (United States); K. Minschwaner, New Mexico Institute of Mining and Technology (United States); S. A. Budzien, U.S. Naval Research Lab. (United States); R. L. Bishop, J. H. Hecht, The Aerospace Corp. (United States)

814805 The RAIDS experiment on the ISS: on-orbit performance [8148-04]

S. A. Budzien, A. W. Stephan, U.S. Naval Research Lab. (United States); R. L. Bishop,

A. B. Christensen, J. H. Hecht, The Aerospace Corp. (United States); K. R. Minschwaner, New Mexico Institute of Mining and Technology (United States)

814806 Characterization of Teledyne microdosimeters for space weather applications [8148-05] C. D. Lindstrom, Air Force Research Lab. (United States); J. D. Sullivan, Boston College (United States); B. K. Dichter, F. A. Hanser, Assurance Technology Corp. (United States); D. Carssow, Air Force Research Lab. (United States); G. E. Galica, Assurance Technology Corp. (United States) 
814807 The Fabry-Pérot interferometer prototype for the ADAHELI solar small mission (Invited Paper) [8148-06]

F. Berrilli, M. Cocciolo, L. Giovannelli, D. Del Moro, F. Giannattasio, R. Piazzesi, M. Stangalini, A. Egidi, Univ. degli Studi di Roma Tor Vergata (Italy); F. Cavallini, INAF - Osservatorio Astrofisico di Arcetri (Italy); V. Greco, Istituto Nazionale di Ottica Applicata, CNR (Italy);

S. Selci, Institute for Complex Systems, CNR (Italy)

814808 Liquid crystals Lyot filter for solar coronagraphy [8148-07]

S. Fineschi, G. Capobianco, G. Massone, INAF - Osservatorio Astronomico di Torino (Italy);

T. Baur, Meadowlark Optics, Inc. (United States); A. Bemporad, L. Abbo, L. Zangrilli, INAF -

Osservatorio Astronomico di Torino (Italy); V. Dadeppo, CNR IFN LUXOR Lab. (Italy)

814809 Ground-based synoptic instrumentation for solar observations (Invited Paper) [81 48-08]

K. S. Balasubramaniam, Air Force Research Lab. (United States); A. Pevtsov, National Solar Observatory (United States)

8148 OA Figure testing and calibration of the ISOON Fabry-Pérot etalons [8148-09]

B. Robinson, The Univ. of Alabama in Huntsville (United States); K. S. Balasubramaniam, Air Force Research Lab. (United States); J. Justice, F. Pitts, ARINC Engineering Services, LLC (United States)

\section{SESSION 3 SOLAR-C}

8148 OB The SOLAR-C mission: current status (Invited Paper) [8148-10]

T. Shimizu, Japan Aerospace Exploration Agency (Japan); S. Tsuneta, H. Hara, National Astronomical Observatory of Japan (Japan); K. Ichimoto, Hida Observatory, Kyoto Univ. (Japan); K. Kusano, Nagoya Univ. (Japan); T. Sakao, Japan Aerospace Exploration Agency (Japan); T. Sekii, Y. Suematsu, T. Watanabe, National Astronomical Observatory of Japan (Japan)

$81480 \mathrm{C}$ Photon-counting soft $\mathrm{x}$-ray telescope for the Solar-C mission [8148-11] T. Sakao, N. Narukage, Japan Aerospace Exploration Agency (Japan); M. Shimojo, S. Tsuneta, Y. Suematsu, S. Miyazaki, National Astronomical Observatory of Japan (Japan); S. Imada, N. Nishizuka, K. Watanabe, T. Dotani, Japan Aerospace Exploration Agency (Japan); E. E. DeLuca, Harvard-Smithsonian Ctr. for Astrophysics (United States);

S. Ishikawa, Univ. of California, Berkeley (United States)

8148 OD Short telescope design of 1.5-m aperture solar UV visible and IR telescope aboard Solar-C [8148-12]

Y. Suematsu, Y. Katsukawa, National Astronomical Observatory of Japan (Japan); T. Shimizu, Japan Aerospace Exploration Agency (Japan); K. Ichimoto, Hida Observatory, Kyoto Univ. (Japan); T. Horiuchi, Y. Matsumoto, PLANET Inc. (Japan); N. Takeyama, Genesia Corp. (Japan)

8148 OE Focal plane instrument for the Solar UV-Vis-IR Telescope aboard SOLAR-C [8148-13] Y. Katsukawa, Y. Suematsu, National Astronomical Observatory of Japan (Japan); T. Shimizu, Japan Aerospace Exploration Agency (Japan); K. Ichimoto, Kwasan and Hida Observatories, Kyoto Univ. (Japan); N. Takeyama, Genesia Corp. (Japan) 
8148 OF Mgll observations using the MSFC solar ultraviolet magnetograph [8148-14] E. West, J. Cirtain, NASA Marshall Space Flight Ctr. (United States); K. Kobayashi, J. Davis, A. Gary, The Univ. of Alabama in Huntsville (United States); M. Adams, NASA Marshall Space Flight Ctr. (United States)

8148 OG Solar EUV Monitor (SEM) absolute irradiance measurements and how they are affected by choice of reference spectrum [8148-15]

S. R. Wieman, D. L. Judge, L. V. Didkovsky, The Univ. of Southern California (United States)

$8148 \mathrm{OH} \quad$ Overview of Chromospheric Lyman-Alpha SpectroPolarimeter (CLASP) [8148-16] N. Narukage, Japan Aerospace Exploration Agency (Japan); S. Tsuneta, T. Bando, R. Kano, M. Kubo, R. Ishikawa, H. Hara, Y. Suematsu, Y. Katsukawa, National Astronomical Observatory of Japan (Japan); H. Watanabe, K. Ichimoto, Kwasan and Hida Observatories, Kyoto Univ. (Japan); T. Sakao, T. Shimizu, Japan Aerospace Exploration Agency (Japan); K. Kobayashi, B. Robinson, The Univ. of Alabama in Huntsville (United States); T. Kim, A. Winebarger, E. West, J. Cirtain, NASA Marshall Space Flight Ctr. (United States); B. De Pontieu, Lockheed Martin Solar \& Astrophysics Lab. (United States); R. Casini, High Altitude Observatory, National Ctr. for Atmospheric Research (United States); J. Trujillo Bueno, J. Stepan, R. Manso Sainz, L. Belluzzi, A. Asensio Ramos, Instituto de Astrofísica de Canarias (Spain); M. Carlsson, Univ. of Oslo (Norway)

8148 OI LEMUR (Large European Module for solar Ultraviolet Research): a VUV imaging spectrograph for the JAXA Solar-C Mission [8148-17]

C. M. Korendyke, U.S. Naval Research Lab. (United States); L. Teriaca, Max-Planck-Institut für Sonnensystemforschung (Germany); G. A. Doschek, U.S. Naval Research Lab. (United States); L. K. Harra, Univ. College London (United Kingdom); U. H. Schühle, Max-Planck-Institut für Sonnensystemforschung (Germany); T. Shimizu, Japan Aerospace Exploration Agency (Japan)

\section{SESSION 5 SOLAR MISSIONS II}

$81480 \mathrm{~J}$ The coronal suprathermal particle explorer (C-SPEX) [8148-18]

J. D. Moses, C. Brown, G. Doschek, Y.-K. Ko, C. Korendyke, J. M. Laming, D. Socker, A. Tylka, U.S. Naval Research Lab. (United States); D. McMullin, Space Systems Research Corp. (United States); C. Ng, George Mason Univ. (United States); S. Wassom, Utah State Univ. (United States); M. Lee, The Univ. of New Hampshire (United States); F. Auchère, Institut d'Astrophysique Spatiale (France); S. Fineschi, INAF - Osservatorio Astronomico di Torino (Italy); T. Carter, Praxis Inc (United States)

8148 OK The Lyman-alpha telescope of the extreme ultraviolet imager on Solar Orbiter [8148-19] U. Schühle, Max-Planck-Institut für Sonnensystemforschung (Germany); J.-P. Halain, Univ. de Liège (Belgium); S. Meining, L. Teriaca, Max-Planck-Institut für Sonnensystemforschung (Germany) 
8148 OM SiC/Mg multilayer coatings for SCORE coronagraph: long term stability analysis (Invited Paper) [8148-21]

M. G. Pelizzo, CNR IFN LUXOR Lab. (Italy) and Univ. degli Studi di Padova (Italy); S. Fineschi, INAF - Osservatorio Astronomico di Torino (Italy); P. Zuppella, A. J. Corso, CNR IFN LUXOR Lab. (Italy) and Univ. degli Studi di Padova (Italy); D. L. Windt, Reflective X-Ray Optics LLC (United States); P. Nicolosi, CNR IFN LUXOR Lab. (Italy) and Univ. degli Studi di Padova (Italy)

8148 ON Development of multilayer thin film filters for the full-sun imager on Solar $O$ rbiter [8148-22] F. Auchère, X. Zhang, Institut d'Astrophysique Spatiale, CNRS, Univ. Paris-Sud 11 (France); F. Delmotte, E. Meltchakov, Lab. Charles Fabry de I'Institut d'Optique, CNRS, Univ. Paris-Sud 11 (France); A. BenMoussa, Royal Observatory of Belgium (Belgium)

$814800 \quad$ High-resolution solar imaging with a photon sieve [8148-23]

J. M. Davila, NASA Goddard Space Flight Ctr. (United States)

8148 OR Spectral features: an overview [8148-26]

$\mathrm{H}$. van Brug, TNO Science and Industry (Netherlands)

8148 OS Atmospheric turbulence and high-precision ground-based solar polarimetry [8148-27] K. Nagaraju, A. Feller, Max-Planck-Institut für Sonnensystemforschung (Germany); S. Ihle, H. Soltau, PNSensor GmbH (Germany)

\section{POSTER SESSION}

8148 OT Ly-alpha polarimeter design for CLASP rocket experiment [8148-28]

H. Watanabe, Kwasan and Hida Observatories, Kyoto Univ. (Japan); N. Narukage, M. Kubo, R. Ishikawa, T. Bando, R. Kano, S. Tsuneta, National Astronomical Observatory of Japan (Japan); K. Kobayashi, The Univ. of Alabama in Huntsville (United States); K. Ichimoto, Kwasan and Hida Observatories, Kyoto Univ. (Japan); J. Trujillo-Bueno, Observatorio del Teide, Instituto de Astrofísica de Canarias (Spain)

8148 OU DIMMI-2h a MOF-based instrument for Solar Satellite ADAHELI [8148-29] M. Stangalini, Univ. degli Studi di Roma Tor Vergata (Italy); P. F. Moretti, Consiglio Nazionale delle Ricerche (Italy); F. Berrilli, D. Del Moro, Univ. degli Studi di Roma Tor Vergata (Italy); S. M. Jefferies, Univ. of Hawai'i (United States); G. Severino, M. Oliviero, INAF - Osservatorio Astronomico di Capodimonte (Italy)

$8148 \mathrm{OV}$ The intensity effect in magneto-optical filters [8148-30] M. Oliviero, G. Severino, INAF - Osservatorio Astronomico di Capodimonte (Italy); F. Berrilli, Univ. degli Studi di Roma Tor Vergata (Italy); P. F. Moretti, Consiglio Nazionale delle Ricerche (Italy); S. M. Jefferies, Univ. of Hawai'i (United States)

8148 OW OPSys: optical payload systems facility for testing space coronagraphs [8148-31] S. Fineschi, G. Crescenzio, G. Massone, G. Capobianco, L. Zangrilli, E. Antonucci, INAF Osservatorio Astronomico di Torino (Italy); F. Anselmi, Alca Technology (Italy)

$81480 \mathrm{X}$ Long term stability of optical coatings in close solar environment [8148-32] A. J. Corso, P. Zuppella, P. Nicolosi, M. G. Pelizzo, CNR IFN LUXOR Lab. (Italy) and Univ. degli Studi di Padova (Italy) 
$81480 Z$ Earth-Affecting Solar Causes Observatory (EASCO): a mission at the Sun-Earth L5 [8148-34] N. Gopalswamy, J. M. Davila, NASA Goddard Space Flight Ctr. (United States); F. Auchère, Institut d'Astrophysique Spatiale, CNRS, Univ. Paris-Sud 11 (France); J. Schou, Stanford Univ. (United States); C. Korendyke, U.S. Naval Research Lab. (United States); A. Shih, NASA Goddard Space Flight Ctr. (United States); J. C. Johnston, Air Force Research Lab. (United States); R. J. MacDowall, NASA Goddard Space Flight Ctr. (United States); M. Maksimovic, LESIA, Univ. de Paris Meudon (France); E. Sittler, A. Szabo, R. Wesenberg, NASA Goddard Space Flight Ctr. (United States); S. Vennerstrom, DTU Space (Denmark); B. Heber, Christian Albrecht Univ. (Germany)

814810 Space-qualified liquid-crystal variable retarders for wide-field-of-view coronagraphs [8148-35]

N. Uribe-Patarroyo, A. Alvarez-Herrero, P. García Parejo, J. Vargas, R. L. Heredero, R. Restrepo, Instituto Nacional de Técnica Aeroespacial (Spain); V. Martínez Pillet, Instituto de Astrofísica de Canarias (Spain); J. C. del Toro Iniesta, A. López, Instituto de Astrofísica de Andalucía (Spain); S. Fineschi, G. Capobianco, INAF - Osservatorio Astronomico di Torino (Italy); M. Georges, Univ. de Liège (Belgium); M. López, Visual Display S.L.L. (Spain); G. Boer, ARCoptix S.A. (Switzerland); I. Manolis, European Space Agency, ESTEC (Netherlands)

Author Index 
Downloaded From: https://www.spiedigitallibrary.org/conference-proceedings-of-spie on 26 Apr 2023

Terms of Use: https://www.spiedigitallibrary.org/terms-of-use 


\title{
Conference Committee
}

\author{
Program Track Chair
}

Oswald H. Siegmund, University of California, Berkeley (United States)

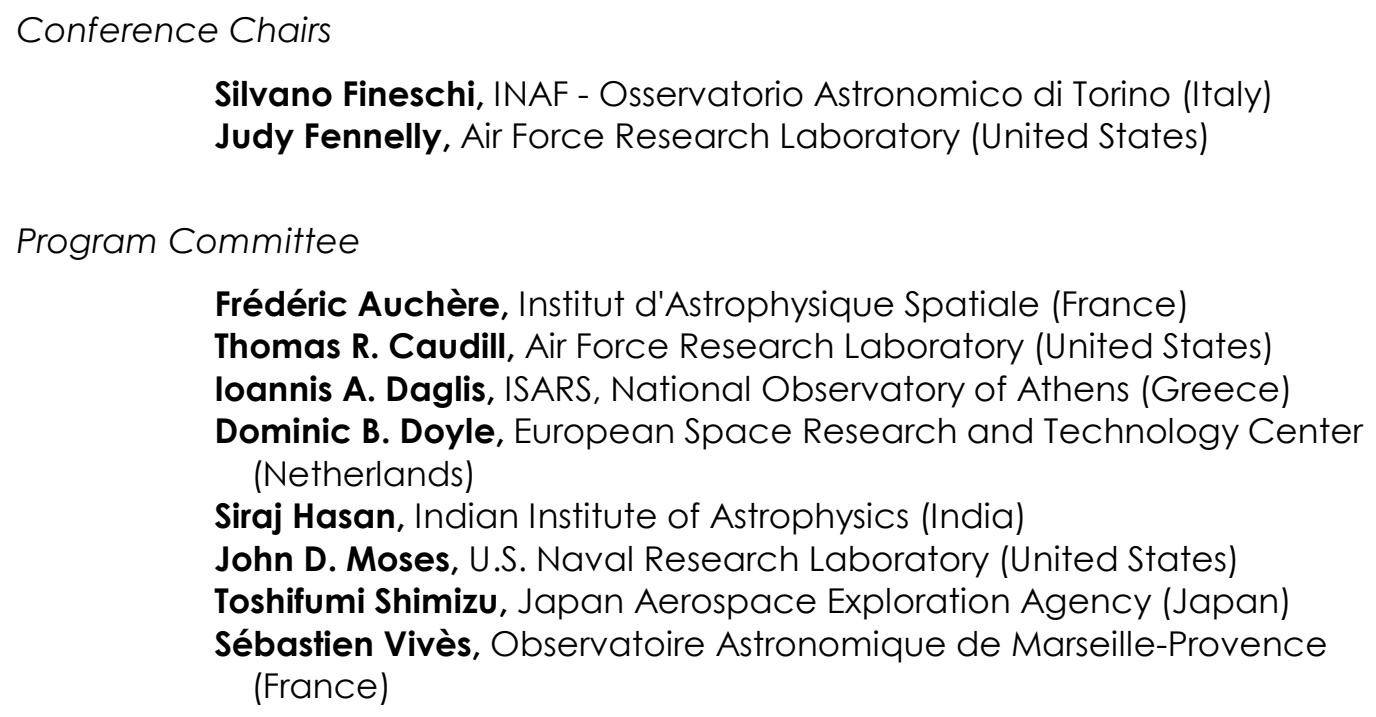

Silvano Fineschi, INAF - Osservatorio Astronomico di Torino (Italy) Judy Fennelly, Air Force Research Laboratory (United States)

Program Committee

Frédéric Auchère, Institut d'Astrophysique Spatiale (France)

Thomas R. Caudill, Air Force Research Laboratory (United States)

loannis A. Daglis, ISARS, National Observatory of Athens (Greece)

Dominic B. Doyle, European Space Research and Technology Center (Netherlands)

Siraj Hasan, Indian Institute of Astrophysics (India)

John D. Moses, U.S. Naval Research Laboratory (United States)

Toshifumi Shimizu, Japan Aerospace Exploration Agency (Japan)

Sébastien Vivès, Observatoire Astronomique de Marseille-Provence

(France)

Session Chairs

1 Space Weather Instrumentation

Judy Fennelly, Air Force Research Laboratory (United States)

2 Imaging Spectroscopy

Erik Wilkinson, Southwest Research Institute (United States)

3 Solar-C

Silvano Fineschi, INAF - Osservatorio Astronomico di Torino (Italy)

4 Solar Missions I

John D. Moses, U.S. Naval Research Laboratory (United States)

5 Solar Missions II

Frédéric Auchère, Institut d'Astrophysique Spatiale (France)

Joseph M. Davila, NASA Goddard Space Flight Center (United States)

6 Advanced Optics for Solar Instrumentation

John D. Moses, U.S. Naval Research Laboratory (United States) 
Downloaded From: https://www.spiedigitallibrary.org/conference-proceedings-of-spie on 26 Apr 2023

Terms of Use: https://www.spiedigitallibrary.org/terms-of-use 